REVISTA DE LA ESCUELA DE CIENCIAS DE LA EdUCACIÓN, AÑo 17, NRO. 16, VOL. 2, JULIO A DiCIEMBRE DE 2021. PÁGINAS 163-170. ISSN 2362-3349 (EN LÍNEA). PRÁCTICAS DE ACOMPAÑAMIENTO Y SEGREGACIÓN EDUCATIVA EN EL INGRESO A ETP-CET PÚBLICOS DE LA CIUDAD DE SAN CARLOS DE BARILOCHE, PROVINCIA DE RÍO NEGRO. VIVIANA ISABEL SEOANE. MARIANA LAURA DELGADO.

\title{
PRÁCTICAS DE ACOMPAÑAMIENTO Y SEGREGACIÓN EDUCATIVA EN EL INGRESO A ETP-CET PÚBLICOS DE LA CIUDAD DE SAN CARLOS DE BARILOCHE, PROVINCIA DE RÍO NEGRO
}

\author{
Viviana Isabel Seoane* \\ Universidad Nacional de La Plata, Argentina \\ viviseoane@gmail.com \\ Mariana Laura Delgado ** \\ Instituto de Formación Docente Bariloche, Argentina \\ marianadelgado@educ.ar
}

Recibido: 20/08/2020 - Aceptado: 6/11/2020

\section{Resumen}

La tesis desarrollada en el marco de la Maestría en Educación de la Facultad de Humanidades y Ciencias de la Educación de la UNLP buscó abordar las formas de regulación estatal y las prácticas docentes de acompañamiento a Ixs estudiantes de primer año de la escuela secundaria técnica, en su relación con la garantía de igualdad de oportunidades en la provincia de Río Negro. El trabajo de campo fue realizado en dos escuelas secundarias de la modalidad técnico profesional de la ciudad de San Carlos de Bariloche durante el año 2018.

En la localidad se observó la persistencia de mecanismos administrativos y burocráticos que, apoyados en la vigencia de un marco regulatorio progresivo, hacen que el Estado regule el ingreso a la escuela secundaria bajo la forma de un sistema igualador que esconde la arbitrariedad que supone el azar (sorteo). Esta regulación impide que las familias y Ixs $^{1}$ jóvenes elijan la escuela, y desconoce la importancia que tiene la escuela en la construcción del proyecto de vida.

Los objetivos que se propuso esta investigación, en términos generales buscaron comprender los modos en que la regulación estatal y las prácticas docentes de acompañamiento en los primeros años de ETP

\footnotetext{
*Profesora en Ciencias de la Educación (FaHCE-UNLP). Magíster en Ciencias Sociales con orientación en Educación y Doctora en Ciencias Sociales (FLACSO/Argentina). Profesora Titular Interina de la Cátedra Política y Legislación de la Educación del Departamento de Ciencias de la Educación (FaHCE-UNLP). Integra el Comité Académico de la Especialización en Educación en Géneros y Sexualidades (FaHCE-UNLP). Docente en carreras de posgrado en educación, géneros y sexualidades. Investigadora del CInIG-IdHCS-UNLP en el área de Política, Educación y Género. Co-dirige el proyecto de investigación Estudios Feministas en Educación: políticas, memorias, prácticas y sujetos en/entre escuelas, universidades y sindicatos (FaHCE-UNLP) y acaba de publicar una compilación junto a Ma. Elena Martínez Derechos humanos, feminismos y educación: interpelaciones y experiencias, Prohistoria, 2020.

** Magister en Educación, FaHCE UNLP. Licenciada en Educación, UNRN. Especialista de Nivel Superior en Educación y TIC, INFD. Diplomada en Ciencias Sociales con mención en Gestión Educativa, Flacso Argentina. Diplomada en Filosofía de la Liberación, FHyCS UNJu. Profesora de Enseñanza Inicial y Primaria, IFDC Bariloche. Expositora en Jornadas, Congresos y Encuentros vinculados a la temática en Argentina. Profesora regular en Práctica Docente del IFD, en Profesorado secundario de Educación Técnica Profesional y Profesorado de Educación Inicial.

${ }^{1}$ Se adopta como convención en este escrito, desde los aportes de Péchin (2013), el uso de la "x" como una reflexión y posicionamiento con respecto a los usos sintácticos de género, en la complejidad de la gramática política del discurso convencional. En este sentido "la " $x$ " intenta cancelar o impugnar la lectura de género, interrumpirlo al menos como binario, marcar el silencio frente a la hipersignificatividad de la declinación femenina y masculina de la lengua castellana".
} 
REVISTA DE LA ESCUELA DE CIENCIAS DE LA EDUCACIÓN, AÑO 17, NRO. 16, VOL. 2, JULIO A DiCIEMBRE DE 2021. PÁGINAS 163-170. ISSN 2362-3349 (EN LÍNEA). PRÁCTICAS DE ACOMPAÑAMIENTO Y SEGREGACIÓN EDUCATIVA EN EL INGRESO A ETP-CET PÚBLICOS DE LA CIUDAD DE SAN CARLOS DE BARILOCHE, PROVINCIA DE RÍO NEGRO. VIVIANA ISABEL SEOANE. MARIANA LAURA DELGADO.

de nivel secundario en Bariloche, afectan o garantizan el principio de igualdad de oportunidades. Asimismo, comprender la relación entre la segregación de la matrícula según sector social con los mecanismos de regulación estatal y las prácticas docentes de acompañamiento que las escuelas ofrecen en los primeros años a Ixs ingresantes.

Palabras clave: Desigualdad - Segregación residencial - Trayectorias escolares - Prácticas docentes Acompañamiento.

\title{
ACCOMPANIMENT PRACTICES AND EDUCATIVE SEGREGATION ON ENTRY TO ETP- CET PUBLIC IN THE CITY OF SAN CARLOS DE BARILOCHE, PROVINCE OF RÍO NEGRO
}

\begin{abstract}
The master's degree thesis of the faculty of humanities and educational science on the UNLP sought to address the actual state regulation ways and the accompanying teaching practices on the first year of secondary technical school, in relation with the guarantee of equal opportunities in Rio Negro province. The field work was carried out in two secondary schools in the technical professional modality on San Carlos de Bariloche city during the 2018

In the locality, the persistence of administrative and bureaucratic mechanisms was observed that, supported by the validity of a progressive regulatory framework, make the state regulate admission to secondary school under the way of an equalizing system that hides the arbitrariness that chance supposes (raffle). This regulation prevents families and young people from choosing school, and ignores the importance of school in the life project construction.

The objectives that this research set out, in general terms, sought to understand the ways in which state regulation and accompanying teaching practices in the first years of secondary education in Bariloche affect or guarantee the principle of equal opportunities. Also understand the relationship between the segregation of enrollment according to social sector with the mechanisms of state regulation and the accompanying teaching practices that schools offer in the early years to new entrants.
\end{abstract}

Keywords: Inequality - Residential segregation - School trajectories - Teaching practices - Accompaniment.

\section{Reseña}

La tesis desarrollada en el marco de la Maestría en Educación de la Facultad de Humanidades y Ciencias de la Educación de la UNLP, titulada "Prácticas de acompañamiento y segregación educativa en el ingreso a ETP-CET públicos de la ciudad de San Carlos de Bariloche, provincia de Río Negro", buscó abordar las formas de regulación estatal que persisten en la actualidad y aquellas prácticas docentes gestadas en primer año de la modalidad técnico profesional, en un marco de obligatoriedad del nivel y en su relación con la garantía de igualdad de oportunidades.

La escuela secundaria en todas sus modalidades se constituye en un derecho para Ixs jóvenes de nuestro país con la sanción de la Ley de Educación Nacional № 26.206/06 que establece la obligatoriedad del nivel. Un año antes, probablemente por la urgencia de reconstituir el circuito de educación técnica que había sido desconfigurado por la reforma educativa inscripta en la Ley Federal de Educación № 24.195/93, se sanciona la Ley de Educación Técnico Profesional $N^{\circ} 26.058$ cuyo objeto es "regular y ordenar la educación técnico profesional en el nivel medio y superior no universitario del SEA y la Formación Profesional".

Además de las dificultades para Ixs jóvenes que supone el ingreso a la escuela secundaria, se agrega a las bajas tasas de terminalidad, el problema de la desigualdad que trae aparejada la segregación educativa. Al respecto se evidencian segregaciones demográficas y residenciales, migración hacia escuelas privadas, aumento de selección de estudiantes por parte de establecimientos a partir de mecanismos basados en la trayectoria educativa- escolar. La sobreedad en los primeros años de la escuela secundaria se constituye en un indicador de las dificultades que encuentran Ixs jóvenes para responder a los requerimientos de una escuela que se les presenta como nueva. Esta regulación impide que las familias y Ixs jóvenes elijan la escuela, y desconoce la importancia que la misma tiene en la construcción del proyecto de vida.

Se trata de una investigación socioeducativa, que adoptó un enfoque preeminentemente etnográfico. Recupera desarrollos de la sociología de las desigualdades en su vinculación con segregaciones socio-residenciales. Retoma la normativa en la modalidad técnico profesional en relación con las trayectorias y el derecho a la escolarización secundaria (Arcidiácono y Otrxs, 2014; Kessler, 2014; Martínez, Villa y Seoane, 2009; Rossetti, 2014).

El enfoque preeminentemente etnográfico, como forma de investigación social, permitió conocer, entender e interpretar en la cotidianidad los sentidos que encierran las voces y las acciones de lxs actores presentes en las instituciones educativas, junto a una encuesta a estudiantes de primer año de dos establecimientos estatales de la 
REVISTA DE LA ESCUELA DE CIENCIAS DE LA EDUCACIÓN, AÑo 17, NRO. 16, VOL. 2, JULIO A DiCIEMBRE DE 2021. PÁGINAS 163-170. ISSN 2362-3349 (EN LÍNEA). PRÁCTICAS DE ACOMPAÑAMIENTO Y SEGREGACIÓN EDUCATIVA EN EL INGRESO A ETP-CET PÚBLICOS DE LA CIUDAD DE SAN CARLOS DE BARILOCHE, PROVINCIA DE RÍO NEGRO. VIVIANA ISABEL SEOANE. MARIANA LAURA DELGADO.

modalidad en la ciudad con orientaciones similares (García, 2015).

Cómo categorías centrales en la tesis se abordan: la segregación socio espacial, prácticas de acompañamiento y las trayectorias educativas-escolares en el ingreso a la escuela secundaria técnica de la modalidad técnico profesional. El desarrollo, composición y tipos de trayectorias, desde los aportes de Terigi (2010), Acosta (2006), Bourdieu (1998), Freixas (2006), Ziegler \& Nobile (2012), como opción metodológica intentan relevar la temporalidad de la experiencia y a la vez posibilitan identificar las diferentes formas de atravesar la experiencia escolar en la escuela secundaria técnica. Otra categoría que recorre esta investigación son las prácticas docentes, como prácticas de acompañamiento.

La tesis se estructura en ocho capítulos, el primero contiene una introducción con la presentación del problema de investigación y descripción metodológica. En capítulos subsiguientes se desarrolla la configuración de la ETP en el país y en la provincia de Río Negro dando lugar a una trama normativa que define la educación secundaria en el marco del derecho a la educación. En un cuarto capítulo se analizan las características socio-demográficas de Ixs estudiantes de primer año de dos Centros de Educación Técnica (CET). El quinto capítulo propone un análisis de los mecanismos de ingreso y las estrategias familiares y el sexto avanza en las prácticas y las trayectorias educativasescolares de segregación en sendos CET. Por último, el capítulo siete indaga en las prácticas de acompañamiento a Ixs estudiantes recién llegadxs a los mencionados centros. Finalmente, en el capítulo ocho, se sintetizan las conclusiones, algunas de las principales afirmaciones y hallazgos volcados a lo largo del trabajo.

La hipótesis de la tesis se vincula a la comprensión de los modos en que la regulación estatal y las prácticas docentes de acompañamiento en los primeros años afectan o garantizan el principio de igualdad de oportunidades de Ixs jóvenes que asisten a la escuela técnica. Caracterizar la configuración histórica de la ETP en la localidad, permitió identificar los criterios de ingreso que sostiene la regulación provincial y las prácticas de acompañamiento que se ponen en marcha frente a estos últimos. En este sentido se tornó fundamental la caracterización socio-económica de la población escolar, su grado de representación en las escuelas seleccionadas en relación con la ampliación de la demanda. De esta manera se buscó problematizar los mecanismos estatales de ingreso a la escuela secundaria y las prácticas de acompañamiento de las trayectorias escolares de Ixs estudiantes, así como la segregación de la oferta educativa, en su vinculación con la igualdad de oportunidades en las trayectorias escolares de Ixs estudiantes.

El recorrido seguido por la investigación titulada "Prácticas de acompañamiento y segregación educativa en el ingreso a CET públicos de Bariloche", partió de la selección de dos establecimientos como son el CET 2 y el CET 28 porque permiten mostrar cómo fue evolucionando la oferta de educación secundaria y particularmente de la modalidad técnico profesional en vinculación con los marcos normativos nacionales y provinciales. En el año 2006 a partir de la sanción de la LEN se establece la obligatoriedad del nivel. Un año antes se sanciona la Ley de Educación Técnico Profesional que buscó regular y reordenar la modalidad. En la provincia de Río Negro en 2008 se sanciona la Ley de Educación Técnico Profesional y en 2012 la Ley Orgánica de Educación y Resoluciones provinciales que regulan en el caso de estas últimas el ingreso a la escuela secundaria.

En la provincia el mecanismo de ingreso a la modalidad es a través de la inscripción en planilla en el último año del nivel primario. En el caso que la demanda de vacantes de un establecimiento supere la oferta, esta es regulada a través de la figura de "sorteo". En la provincia existen 37 establecimientos de la modalidad (32 estatales y 5 privados), mientras que la localidad cuenta con 6 establecimientos de los cuales 3 son estatales y 3 de gestión privada/gestión social. Eso convierte a Bariloche en la localidad de la provincia que más establecimientos privados posee. La persistencia de mecanismos administrativos y burocráticos, apoyados en la vigencia de un marco regulatorio progresivo, hacen que el Estado regule el ingreso a la escuela secundaria bajo la forma de un sistema igualador que esconde la arbitrariedad que supone el azar (sorteo). Esta regulación impide que las familias y Ixs jóvenes elijan la escuela y desconoce la importancia que tiene esta última en la construcción del proyecto de vida. Estamos ante una respuesta política para resolver la demanda, en base al rendimiento de las trayectorias y el origen social, que operaría negativamente en las trayectorias y oportunidades educativas de Ixs jóvenes.

Este estudio indaga en qué medida la regulación del acceso a la ETP secundaria refuerza la segregación educativa por razones geográficas y sociales; si las prácticas de acompañamiento facilitan la transición de un nivel a otro y el tránsito por la ETP. Además, si se ofrecen mejores oportunidades para que Ixs estudiantes alcancen una trayectoria exitosa, luego de haber sido expuestos a los efectos de un sistema de ingreso que se presenta como inexorable y que decide por las familias y Ixs jóvenes a cuál escuela técnica asistir y qué especialidad estudiar.

En el capítulo 2 se recuperan los orígenes de la escuela secundaria en Argentina y los marcos normativos que se fueron gestando en diversas épocas. La escuela secundaria en sus orígenes estuvo signada por una formación humanística orientada a la consolidación de la burocracia estatal (Bottinelli \& Sleiman, 2015). En el caso de la ETP, tuvo una matriz ligada a sectores populares en ascenso, en un contexto de modernización del aparato productivo. Será 1939 con la demanda del Movimiento Obrero y la burguesía industrial, el momento de creación de un circuito de enseñanza técnico profesional por parte del Estado de amplia cobertura y acceso (Balduzzi, 1987; Cucuzza, 1994). Lo hará bajo una matriz "androcéntrica, capitalista y en ocasiones homofóbica" que incorpora a las mujeres en los márgenes de la modalidad (Seoane, 2013). Posteriormente los gobiernos pos peronistas pondrán a la modalidad en los "bordes del sistema"; en los 80 se impulsará un sistema dual y en los 90 se dará cierre a las opciones técnicas (Pineau, 1997)

A partir del año 2005 se comenzará a reconstituir el tejido de la modalidad con la sanción de la Ley de Educación Técnica. En Río Negro en 2008 se sanciona la Ley de Educación Técnico Profesional y la resolución que 
REVISTA DE LA ESCUELA DE CIENCIAS DE LA EDUCACIÓN, AÑO 17, NRO. 16, VOL. 2, JULIO A DiCIEMBRE DE 2021. PÁGINAS 163-170. ISSN 2362-3349 (EN LÍNEA). PRÁCTICAS DE ACOMPAÑAMIENTO Y SEGREGACIÓN EDUCATIVA EN EL INGRESO A ETP-CET PÚBLICOS DE LA CIUDAD DE SAN CARLOS DE BARILOCHE, PROVINCIA DE RÍO NEGRO. VIVIANA ISABEL SEOANE. MARIANA LAURA DELGADO.

reúne a todos los establecimientos bajo la denominación de Centros de Educación Técnica (CET). La matrícula escolar en Argentina muestra una fuerte "tendencia privatizadora" que asciende a más del $25 \%$ para el nivel. En Río Negro esta tendencia ha aumentado en todos los niveles desde 1998, alcanzando en 2015 más del 20\%. Particularmente en Bariloche, la tendencia privatizadora es pronunciada y en este sentido cuenta con mayor cantidad de escuelas técnicas para el nivel igualando la oferta estatal (Duhalde \& Feldfeber, 2016).

En el capítulo 3 se aborda la configuración de la oferta de Educación técnica en Bariloche. Actualmente existen seis establecimientos, de los cuales tres pertenecen a una oferta pública. La institución de ETP más antigua de la localidad data de 1953, actual CET 2, denominada en sus orígenes ENET 1, ubicada en cercanías del centro de la ciudad. Recién en el año 2011 el Centro de Formación Profesional implementará un plan de estudio para el nivel y la modalidad que lo llevará a llamarse CET 25, el segundo establecimiento técnico de la ciudad. Así por más de cinco décadas el CET 2 se constituyó en la única oferta ETP de la ciudad y recién en el año 2014 se creará la tercera oferta técnica secundaria bajo la denominación de CET 28.

La investigación retoma los estudios de Jara y Blanco (2013) que mostrarán que en la provincia de Río Negro en el año 86 se dará inició a un proceso de reforma del nivel secundario, que no contemplará a la modalidad, pero que reorganizará el nivel con fuerte participación de la comunidad y cambio del formato tradicional. Dicha reforma será derogada en los 90, con fuerte resistencia de docentes, estudiantes y comunidad en general, como también sucederá en las escuelas técnicas que iniciarán un proceso de transferencia final de Nación a la provincia denominándose, sin importar la modalidad, Centros de Educación Media (CEM). En este marco la ENET 1 de Bariloche, único establecimiento técnico en la localidad, pasará a denominarse CEM 51. Será recién en el año 2008 cuando la provincia tomará la decisión de designar Centros de Educación Técnica (CET) a los establecimientos de la modalidad de nivel secundario, y el CEM 51 pasará a llamarse CET 2.

El otro establecimiento que existe en la ciudad, con orientación similar al CET 2, por lo cual fue tomado para el estudio, es el CET 28, localizado en lejanías del centro de la ciudad. Encuentra su creación en la alta demanda de establecimientos técnicos en los últimos años en la localidad. Este establecimiento no contará con edificio propio, hasta julio de 2020, por lo cual desarrollará sus actividades en una escuela primaria y los talleres en un establecimiento público de gestión social. La falta de edificio se constituirá, según la voz de Ixs actores entrevistadxs (preceptoras, maestra fortalecedora MF, director) en un problema ligado a la convivencia entre niveles (primario y secundario), así como la incomodidad por la falta de espacios (pasillos para recreos, talleres en otros edificios, baños compartidos con la escuela primaria, falta de biblioteca propia, entre otros), y como consecuencia, la supuesta no elección en primer orden para muchxs estudiantes cuando se realiza la inscripción a primer año. También se buscó mostrar desde la voz de Ixs actores entrevistados del CET 28, en qué medida su creación permitió "mitigar el ingreso al CET 2" y la fuerza que posee este último en su tradición. Pero además una preferencia en la elección ligada a la vinculación que la institución posee con al polo tecnológico local, a través de la presencia de convenios de prácticas profesionalizantes con empresas y organismos cómo CNEA, Invap, ALTEC, entre otras.

En el capítulo 4 se abordaron las características socio demográficas de Ixs estudiantes de primer año del CET 28 y CET 2 y de sus familias a partir de clasificaciones ligadas a sexo género, edades, tipos de hogares, niveles de hacinamiento en el hogar, situación socioeconómica, sostén económico del hogar, tipos de trabajo, barrios de origen e instituciones escolares primarias a las que concurrieron. Los datos mostraron la presencia mayoritaria de varones en ambos establecimientos, la edad de predominio en ambos fue de 13 años y sobreedad en el CET 28 mayoritariamente. El porcentaje de hogares con núcleos conyugales completos fue alto en ambos establecimientos y los niveles de hacinamiento similares en ambas instituciones. En relación al sostén económico del hogar, en el CET 2 la presencia masculina fue mayor que en el otro establecimiento, así como actividades laborales de tipo permanente prevalecieron en el primero. En ambas instituciones la tercera parte de Ixs estudiantes manifestó ser aportante bajo la figura de "ayuda familiar" y "changa".

Al analizar los barrios de origen de estudiantes de primer año en ambos centros, quienes concurrían al CET 28 pertenecían a la tipología de agrupamiento de barrios "populares suburbanos", con baja presencia en barrios de "viviendas sociales"; caracterizados en su mayoría por bajo nivel socioeducativo e infraestructura y servicios consolidados sólo en la mitad. Un importante grupo habita barrios con "restricciones de infraestructura y muy bajo nivel socioeconómico". En el CET 2 se modifica sustancialmente la tipología de barrios, con significativa mejora de la infraestructura, servicios, sectores asalariados y buenos niveles educativos de Ixs adultxs. Además, este establecimiento presentó agrupamientos que no tienen representación en el CET 28, como son "barrios céntricos" y quiénes habitan en el "oeste de la ciudad" con consolidación en materia de infraestructura y servicios, sectores asalariados y buenos niveles educativos. El porcentaje de estudiantes varones del CET 2 cuyas familias tuvieron acceso a viviendas sociales duplica el observado en CET 28 (Niembro, Guevara, Cavanagh, 2018).

En el capítulo 5 se analiza el mecanismo de ingreso a la escuela secundaria en la provincia, que en la ETP lo constituye el sorteo. Existe también la opción de permuta entre familias la cual fue utilizada en ambos establecimientos para el ingreso, tal como arroja la encuesta realizada a estudiantes de primer año, con porcentajes bajos al igual que el ingreso directo por hermanxs o familiares que trabajan en el establecimiento. La responsabilidad del acompañamiento en la elección del establecimiento deseado se encuentra regulada por una norma específica ${ }^{2}$, que recae en docentes y Equipos Técnicos como parte de la labor de asesoramiento. Al analizar el mecanismo de

\footnotetext{
${ }^{2}$ Resolución 5534/04
} 
REVISTA DE LA ESCUELA DE CIENCIAS DE LA EDUCACIÓN, AÑo 17, NRO. 16, VOL. 2, JULIO A DiCIEMBRE DE 2021. PÁGINAS 163-170. ISSN 2362-3349 (EN LÍNEA). PRÁCTICAS DE ACOMPAÑAMIENTO Y SEGREGACIÓN EDUCATIVA EN EL INGRESO A ETP-CET PÚBLICOS DE LA CIUDAD DE SAN CARLOS DE BARILOCHE, PROVINCIA DE RÍO NEGRO. VIVIANA ISABEL SEOANE. MARIANA LAURA DELGADO.

ingreso según sexo género, se observó que alrededor de la mitad de las mujeres del CET 2 lo hicieron bajo la figura de sorteo, mientras que para el CET 28 la cifra fue mucho mayor. Cabe destacar que la variable residencial en las reglamentaciones de ingreso a la secundaria no es considerada, como sí ocurre en otros niveles. Llamó la atención que Ixs estudiantes del CET 28 en su totalidad pertenecían al barrio del establecimiento o habitaban en barrios cercanos. Por otra parte, era reducido el grupo de estudiantes que residía en el barrio del CET 28 y concurría al CET 2, así como estudiantes que habitaban en zonas linderas al CET 2 pero no elegían concurrir al CET 28.

Otro tema que buscó abordar el capítulo han sido los motivos de elección de la ETP, mostrando la importancia dada por Ixs estudiantes a la idea que "prepara mejor para seguir carreras técnicas", opción acentuada en el CET 2. También la influencia de Ixs adultxs en la elección del establecimiento escolar y el conocimiento sobre este, en vinculación con la inserción que los familiares habían tenido en la modalidad; cuestión también abordada en igual sentido por Ixs actores institucionales entrevistadxs. EI CET 2 arrojó una mayor presencia de padres-varones respecto del CET 28 que habían estudiado en la modalidad. En relación a las motivaciones para la elección de la ETP la misma fue dada por la "mejor preparación para el mundo del trabajo", pero también la "sugerencia familiar", triplicadas en el CET 28 respecto al CET 2. En el CET 28 la mitad de estudiantes mujeres ingresó por "sugerencia familiar" mientras que en el CET 2 fue menor.

No terminó de delinearse con claridad en la respuesta privilegiada por estudiantes, si la "preparación para el mundo del trabajo" formaba parte de los propios deseos o se inscribía en demandas adultas. Fueron elocuentes algunas expresiones de estudiantes tales como "deja poco tiempo libre", "no da vida", "cansancio", "sacrificio". Es de suponer que la modalidad técnica, a diferencia del otro formato de escuela secundaria que posee la provincia, con una carga horaria significativa produzca ese malestar entre Ixs estudiantes. Sin embargo, estas consideraciones respecto del tiempo que insume la escuela fueron menores en el CET 28 tal vez por el hecho que Ixs estudiantes realizaron su escolaridad primaria en instituciones de jornada extendida y completa.

El capítulo 6 recupera la experiencia escolar en el ingreso y su incidencia en la trayectoria en vinculación con una oferta segregada de CET en la localidad. Al mismo tiempo se han buscado poner en tensión los discursos que refieren a la exclusiva responsabilidad de estudiantes y familias en las trayectorias, para colocar al Estado como responsable del derecho a la educación. Se consideró la repitencia para el análisis del fenómeno de la segregación educativa a partir del cambio de escuela, edades, interrupción temporaria de la escolaridad, barrios, organización del tiempo extraescolar y prácticas culturales de Ixs jóvenes.

Se observó que la "trayectoria ideal o regular" era alta en ambos establecimientos, siendo mayor en el CET 2, mientras que las trayectorias irregulares eran mayores en CET 28. En ambas instituciones un grupo reducido de estudiantes vio interrumpida su escolaridad durante la primaria; dato que se duplicaba en las mujeres del CET 28. La sobreedad se mostró mayor en CET 28, duplicando al CET 2. En relación a la misma Ixs agentes, cuya función se vincula al acompañamiento, expresaron como causas de la repitencia "falta de estudio", "desinterés" y las "decisiones familiares sobre la modalidad". Todas referencias que ubican la responsabilidad sobre los procesos de escolarización en Ixs estudiantes y las familias.

En torno a la organización del tiempo escolar y las prácticas culturales el estudio evidenció que las tareas que realizaban habitualmente y a las que dedicaban más tiempo eran similares en ambos establecimientos, todas ellas ligadas al uso de celular, así como deportes y escuchar música. El cuidado de hermanxs sólo apareció en estudiantes del CET 28 que, según la maestra fortalecedora era uno de los motivos principales de inasistencia y llegadas tarde. Por el contrario, lectura, aprendizaje de idiomas y "estar con amigxs" fueron actividades exclusivas de estudiantes del CET 2.

En este capítulo se retomó la oferta segregada de la modalidad en la ciudad en su vinculación con la "zonificación" de establecimientos distribuidos desigualmente para Ixs jóvenes, junto a la "aglomeración geográfica de familias de una misma condición o categoría social", como proceso que muestra cierta ineficacia de las políticas de Estado en torno al desarrollo equitativo y de calidad en el acceso a la secundaria, en particular a la modalidad técnica. La "zonificación" refuerza la búsqueda de circuitos escolares de mayor "prestigio" y "deseabilidad colectiva". En este sentido se observa que Ixs estudiantes que concurrían al CET 2 provenían de diversos barrios, haciendo uso de medios de transporte variados; mientras que los del otro establecimiento pertenecían al barrio y zonas aledañas (Rossetti, 2014).

La "deseabilidad colectiva" que representa el CET 2, en la valoración positiva que hace la comunidad sobre el edificio propio, el contexto social, la historia institucional, la buena relación entre los "padres y el nivel académico", se contrapone con un CET 28 "sin historia", presentado por lxs diversos actores institucionales como un establecimiento en un "barrio complicado", "dificultades de bullying y de Ixs estudiantes para poder acceder a los contenidos", junto a una proyección del equipo directivo que plantea la necesidad de pensar orientaciones que posean "un grado de abstracción menor".

El capítulo 7 brinda tratamiento a aquellas figuras de acompañamiento en la escuela a través de "estrategias personalizadas" con significativas diferencias en dichas prácticas para uno y otro establecimiento. Los desempeños profesionales en Río Negro son regulados por resoluciones que dan cuenta de las funciones de preceptores, así como la implementación desde 2012, de un Programa de Acompañamiento al Alumno de Primer Año. A partir del año 2004 comienzan a delinear nuevas institucionalidades para preceptores, como garantía del derecho a la educación secundaria obligatoria. Para el caso del desempeño de la maestra fortalecedora, predomina la ausencia de regulación de la función, a lo que se suma la inestabilidad laboral (Ziegler \& Nobile, 2012). 
REVISTA DE LA ESCUELA DE CIENCIAS DE LA EDUCACIÓN, AÑO 17, NRO. 16, VOL. 2, JULIO A DiCIEMBRE DE 2021. PÁGINAS 163-170. ISSN 2362-3349 (EN LÍNEA). PRÁCTICAS DE ACOMPAÑAMIENTO Y SEGREGACIÓN EDUCATIVA EN EL INGRESO A ETP-CET PÚBLICOS DE LA CIUDAD DE SAN CARLOS DE BARILOCHE, PROVINCIA DE RÍO NEGRO. VIVIANA ISABEL SEOANE. MARIANA LAURA DELGADO.

En la encuesta realizada, Ixs estudiantes reconocen las figuras de estxs actorxs en la trama de sostenimiento de sus trayectorias, pero encuentran mayor protagonismo en profesores, compañerxs y otrxs. Por otra parte, en CET 28, ubican en forma privilegiada a las madres y dan cuenta en las encuestas que conocen la figura de MF, habiéndole consultado en más de una oportunidad. Para el caso de preceptorxs, el reconocimiento como figura de acompañamiento fue bajo, en ambos establecimientos. Al indagar sobre la ayuda recibida por preceptores y MF en CET 2, refieren a un proceso de acompañamiento de fuerte corte "cognitivo-disciplinar". Mientras que en el CET 28 tiene una fuerte presencia lo "vincular" porque si bien el "estudio" prevalece en la mitad de Ixs estudiantes, también lo hacen las intervenciones ligadas a la relación con compañerxs y cuestiones familiares (Ziegler \& Nobile, 2012).

En el marco de políticas de cuidado a las trayectorias escolares Ixs preceptores advierten que su permanencia horaria, bajo variadas formas, se tornan puntos sensibles y particulares en la tarea de acompañamiento. En el CET 2 se presentó la tensión entre "estar en la escuela", pero no lo suficiente, dado que si bien la permanencia diaria era total, el encuentro con Ixs estudiantes se limitaba al inicio de la jornada, en el regreso de los recreos y en la organización de ciertas actividades esporádicas producidas por otrxs. Caracterizaron las horas libres como un "acompañamiento en la emergencia", reconocieron tareas que consideraban no corresponderse con el rol, así como la responsabilidad por la trayectoria escolar ubicada exclusivamente en las familias. Cuestiones que dan cuenta de la centralidad que adquiere el "eje administrativo", así como identificación de ejes de "acompañamiento" y de tratamiento de lo "emergente e indeterminado" en un segundo plano de manera más acentuada en el CET 2 (Niedzwiecki, 2015).

En el caso de las MF, frente a la ausencia de marco regulatorio de la actividad profesional, se observaron diversas prácticas en las que manifestaban la preocupación y la importancia del "sostenimiento". Sin embargo, en el CET 2 la MF brinda a las prácticas de acompañamiento la prevalencia de un "corte cognitivo disciplinar" con acompañamiento en el aula, frente a requerimientos puntuales de docentes. En el CET 28, se vinculan a propuestas que podrían denominarse más amplias respecto al CET 2, dado que trascienden el aula (en biblioteca de primaria, en otros espacios) y las tareas no se limitan a primer año (también a segundo); traduciéndose en acciones de acompañamiento a estudiantes en pequeños grupos, dentro y fuera del aula, sistemáticamente, para el tratamiento de disciplinas específicas, con la presencia de otrxs actores adultxs de la institución.

\section{A modo de cierre}

Esta investigación buscó abordar la segregación demográfica y residencial en su relación con el mecanismo de ingreso a la escuela secundaria de la modalidad ETP en la localidad de Bariloche, provincia de Río Negro, así como aquellas prácticas de acompañamiento, sus lógicas, funciones, desempeños traducidos en construcciones en las instituciones y los marcos normativos que las regulan, durante el primer año en el pasaje de la escuela primaria la secundaria.

En este sentido, buscó analizar, desde los procesos de zonificación que devienen en una distribución desigual de Ixs jóvenes junto a la consolidación de circuitos de escolarización diferenciados, la vinculación con las políticas educativas, las cuales tenderían a reforzar dicha distribución de establecimientos para Ixs estudiantes. En relación a los barrios de procedencia se pudo observar en uno de los establecimientos la ausencia de estudiantes procedentes de "zonas céntricas", "oeste" de la ciudad, junto a la marcada presencia y representación del tipo de agrupación "populares suburbanos", (siguiendo la zonificación propuesta por Niembro, Guevara y Cavanagh, 2018) que se caracteriza por contar con niveles bajos de infraestructura y escasa escolaridad de adultxs, así como tareas del hogar y cuidados acentuadas en Ixs estudiantes, sobre todo mujeres de CET 28, lo cual constituye claramente un obstáculo a su escolaridad plena y al derecho que les asiste.

La segregación residencial y los procesos de zonificación en la localidad conllevan la existencia de prácticas diferenciadas en instituciones con mayores grados de "deseabilidad colectiva", donde se presenta un otorgamiento mayor en torno a la "distinción entre la conducta entre pares", el supuesto basado en el "nivel académico", pero también la "existencia del edificio", entre otros. Algunos de estos presupuestos mostrarían y en algún sentido reforzarían la idea de que el contexto social, económico y cultural "hace la diferencia". Donde la demanda de vacantes en la localidad, excede la oferta existente, y no permite a Ixs estudiantes y sus familias elegir el establecimiento deseado, como proyecto de vida y derecho.

Esta investigación permitió analizar los mecanismos de ingreso a la escuela secundaria en tanto política pública y su relación con las trayectorias educativas de Ixs jóvenes. Respecto de las respuestas que ha dado el Estado rionegrino para atender a la creciente demanda por Educación Técnica hacia los mecanismos que regulan en la provincia y en Bariloche el ingreso, constituye el sorteo, bajo la forma de un sistema que se presenta como igualador, un impedimento para que las familias y Ixs jóvenes elijan el establecimiento. Al mismo tiempo desconoce la importancia en el proyecto de vida, donde el azar-sorteo se presenta como un obstáculo al fortalecimiento necesario del vínculo de Ixs jóvenes con el proceso de escolarización y con la institución de referencia. Asimismo, las experiencias de Ixs estudiantes ante los mecanismos de ingreso que pudimos relevar ponen en tensión las respuestas dadas por el Estado provincial ante la creciente demanda de familias y jóvenes por acceder a la modalidad técnico profesional, y colocan un fuerte interrogante en torno de si el derecho a la educación y la obligatoriedad del nivel son suficientes definiciones políticas para garantizar la igualdad de oportunidades para todxs, o si son necesarias otras mediaciones, prácticas y propuestas que incluyan las visiones y expectativas de Ixs jóvenes.

Para finalizar, resulta fundamental advertir que los marcos normativos que regulan la Escuela Secundaria Rionegrina (ESRN) permiten una organización curricular e institucional que rompe con el formato asentado en las 
REVISTA DE LA ESCUELA DE CIENCIAS DE LA EdUCACIÓN, AÑo 17, NRO. 16, VOL. 2, JULIO A DiCIEMBRE DE 2021. PÁGINAS 163-170. ISSN 2362-3349 (EN LÍNEA). PRÁCTICAS DE ACOMPAÑAMIENTO Y SEGREGACIÓN EDUCATIVA EN EL INGRESO A ETP-CET PÚBLICOS DE LA CIUDAD DE SAN CARLOS DE BARILOCHE, PROVINCIA DE RÍO NEGRO. VIVIANA ISABEL SEOANE. MARIANA LAURA DELGADO.

disciplinas escolares y pone recursos y dispositivos dedicados a Ixs estudiantes que cursan los primeros años, frente a una Educación Técnico Profesional (ETP) que si bien posse funciones tutoriales para los primeros años, su organización es fuertemente disciplinar y no ha estado atravesada por propuestas de reforma. Por último, considero que es importante recuperar del conjunto de reformas aplicadas a la escuela secundaria en la provincia la que refiere a los regímenes de contratación de Ixs Maestrxs Fortalecedorxs si efectivamente se busca en la ETP garantizar políticas de cuidado que se traduzcan en prácticas de acompañamiento a Ixs estudiantes de los primeros de las escuelas técnicas.

\section{Referencias bibliográficas}

Acosta, F. (2009). Re-configuraciòn de la escuela secundaria frente al ingreso masivo de jóvenes de sectores populares. Revista de Pedagogía, 30(87), pp.217-246.

Arcidiácono, M. et. al. (2014). La segregación escolar público-privada en América Latina. Santiago de Chile: CEPAL. Serie Políticas Sociales.

Balduzzi, J. (1987). Peronismo, saber y poder. En: Puiggros, A. et al. (comp.) Hacia una pedagogía de la imaginación para América Latina. Buenos Aires: Contrapunto.

Bottinelli, L. y Sleiman, C. (2015). La educación técnica en Argentina. Buenos Aires: UNIPE

Cucuzza, H. (dir.) El peronismo y la Universidad Obrera Nacional. En: Estrategias político educativas populistas durante la primera mitad del S. XX: Perón-Vargas-Cárdenas. Programa de incentivos del Ministerio de Cultura y Educación 1994/1996. Luján: UNLU, Mimeo.

Duhalde, M. y Feldfeber, M. (2016). Tendencias privatizadoras "de" y "en" la educación argentina. Buenos Aires: CTERA

Freixas, J. (2006). Bienvenir, cuidar, acompañar. La educación como hospitalidad. En: Kohan, W. (comp.) Teoría y práctica en filosofía con niños y jóvenes. Buenos Aires: Novedades educativas. Karla.

García, M. (2015). Itinerarios y operaciones de Análisis del Discurso e Investigación Social. En: Formación de Posgrado. Métodos cualitativos para la Investigación Social contemporánea: técnicas y claves en etnografía y análisis del discurso. Buenos Aires: IDES

Jara, M. y Blanco, L. (2013). El lugar de la historia en los Diseños Curriculares de la provincia de Río Negro. Entre Reformas y Contrarreformas. En: Reseñas № 4 Revista de APEHUN (Asociación de Profesores de Enseñanza de la Historia de Universidades Nacionales). Octubre de 2013. Córdoba: Editorial Universitas, pp. 191. 225.

Kessler, G. (2014). Tendencias contrapuestas en educación, salud y vivienda. En: Controversias sobre la desigualdad. Buenos Aires: Fondo de Cultura Económica, pp. 115. 143.

Martínez, M.; Villa, A. y Seoane, V. (2009). Manteniendo la distancia: mercado escolar y desigualdad social. En: Martínez, M. E.; Villa, A. y Seoane, V. (comp.) Jóvenes, elección escolar y distinción social: investigaciones en la Argentina y Brasil. Buenos Aires: Prometeo

Niembro, A.; Guevara, T. y Cavanagh, E. (2018). Segregación socio-residencial e infraestructura en América Latina: Una tipología de los barrios de Bariloche, Argentina. Bariloche: inédito.

Niedzwiecki, D. (2015). Desde el rincón de las hornallas. La gestión de lo cotidiano en escuelas secundarias de la Ciudad Autónoma de Buenos Aires desde la perspectiva de los preceptores (2013-2014). Buenos Aires: Flacso. Programa de Doctorado en Ciencias Sociales.

Péchin, J. (2013). De la indicación de "perversiones" por parte de la(s) norma(s) a la "perversión" política de la (a) normalización: ¿sujetxs de deseo como sujetxs de derecho? En: LES Online, Vol. 5, $\mathrm{N}^{\circ} 1$. Disponible enhttps://lesonlinesite.files.wordpress.com/2017/03/sujetxs-de-deseo-como-sujetxs-de-derecho.pdf Consultado en abril de 2019

Pineau, P. (1997). La vergüenza de haber sido y el dolor de ya no ser: los avatares de la educación técnica entre 1955 y 1983. En: Puiggros, A. (Dir.) Dictaduras y utopías en la historia reciente de la educación argentina (1955-1983). Buenos Aires: Galerna.

Rossetti, M. (2014). La segregación escolar como un elemento clave en la reproducción de la desigualdad. Santiago de Chile: CEPAL. Serie Políticas Sociales

Seoane, V. (2013). Géneros, cuerpos y sexualidades. Experiencias de Mujeres en Escuelas Técnicas de la ciudad de La Plata. Buenos Aires: FLACSO. Programa de Doctorado en Ciencias Sociales.

Terigi, F. (2010). Las cronologias de aprendizaje, un concepto para pensar las cronologias escolares. Conferencia Apertura Ciclo Lectivo 2010. Santa Rosa: Ministerio de Cultura y Educaciòn

Ziegler, S. y Nobile, M. (2012). Personalización y escuela secundaria: dinámicas de escolarización en diferentes grupos sociales. En: Archivos de Ciencias de la Educación Nro.6. La Plata.

\section{Documentos consultados}

Dirección de Planeamiento y Formación Superior de Río Negro (2014) "La repitencia en nivel secundario. Ciclo Básico Común: CEM y CET 2013. Análisis de la repitencia en el Ciclo Básico del Nivel Secundario. Sector estatal. Comportamiento de la desaprobación de asignaturas del Ciclo Básico y su relación con la Formación Docente". Viedma: Investigación y Estadística Educativa.

Indicadores educativos. Tasa de eficiencia interna. Porcentaje de alumnos con sobreedad. Desagregados por nivel de enseñanza y jurisdicción. Diniee. Ministerio de Educación y Deportes de la Nación.

Ley Federal de Educación N²4195/93

Ley Nacional de Educación N²6206/06

Ley de Educación Técnico Profesional N²6058/05

Ley de Educación Técnico Profesional de Río Negro º4347/08

Ley Orgánica de Educación de Río Negro №2444/92

Ley Orgánica de Educación de Río Negro №4819/12

Resolución provincial 4617/17 Anexo I - Régimen Académico ESRN

Resolución provincial 3360/16 Reglamento ingreso, permanencia de estudiantes a establecimientos secundarios. Anexo I

Resolución provincial 2390/14 Programa de articulación entre nivel primario y nivel secundario 
REVISTA DE LA ESCUELA DE CIENCIAS DE LA EdUCACIÓN, AÑo 17, NRO. 16, VOL. 2, JULIO A DiCIEMBRE DE 2021. PÁGINAS 163-170. ISSN 2362-3349 (EN LÍNEA). PRÁCTICAS DE ACOMPAÑAMIENTO Y SEGREGACIÓN EDUCATIVA EN EL INGRESO A ETP-CET PÚBLICOS DE LA CIUDAD DE SAN CARLOS DE BARILOCHE, PROVINCIA DE RÍO NEGRO. VIVIANA ISABEL SEOANE. MARIANA LAURA DELGADO.

Resolución provincial 137/13 Anexo Planes de Estudio ETP (Ciclo Básico y Superior)

Resolución provincial 1568/13 Programa de Acompañamiento al Alumno de Primer Año

Registro Federal de Instituciones de Educación Técnico Profesional - INET (2012)

Sistema Nacional de Indicadores Educativos. Laboratorio de Estadística. Red Federal de Información Educativa. DINIECE. Ministerio de Educación, Ciencia y Tecnología (2005)

Resolución provincial 310/11 Modificación de Centro de Formación Profesional №1 a Centro de Educación Técnica №25 e implementación de Plan de Estudio correspondiente al Ciclo Básico de Escuelas Técnicas de Nivel Medio, Especialidad Hotelería y Gastronomía

Resolución provincial 946/08 Planes de Estudio Escuelas Técnicas de Río Negro

Resolución provincial 2262/07 Legajo Único de Alumno

Resolución provincial 5534/04 Normas de Asignación de cargos docentes, misiones y funciones en Establecimientos de Nivel Medio del Consejo Provincial de Educación

Informe de Sustentabilidad INVAP 2013- 2015.San Carlos de Bariloche 